\title{
Improved Formulae for the Inductance of Straight Wires
}

\author{
H. A. Aebischer ${ }^{1}$, B. Aebischer ${ }^{2}$ \\ ${ }^{1}$ LEGIC Identsystems AG, Switzerland \\ ${ }^{2}$ Hexagon Technology Center GmbH, Switzerland \\ *corresponding author, E-mail: hubert. aebischer@legic.com
}

\begin{abstract}
The best analytical formulae for the self-inductance of rectangular coils of circular cross section available in the literature were derived from formulae for the partial inductance of straight wires, which, in turn, are based on the well-known formula for the mutual inductance of parallel current filaments, and on the exact value of the geometric mean distance (GMD) for integrating the mutual inductance formula over the cross section of the wire. But in this way, only one term of the mutual inductance formula is integrated, whereas it contains also other terms. In the formulae found in the literature, these other terms are either completely neglected, or their integral is only coarsely approximated. We prove that these other terms can be accurately integrated by using the arithmetic mean distance (AMD) and the arithmetic mean square distance (AMSD) of the wire cross section. We present general formulae for the partial and mutual inductance of straight wires of any cross section and for any frequency based on the use of the GMD, AMD, and AMSD.

Since partial inductance of single wires cannot be measured, the errors of the analytical approximations are computed with the help of exact computations of the six-dimensional integral defining induction. These are obtained by means of a coordinate transformation that reduces the sixdimensional integral to a three-dimensional one, which is then solved numerically. We give examples of an application of our analytical formulae to the calculation of the inductance of short-circuited two-wire lines. The new formulae show a substantial improvement in accuracy for short wires.
\end{abstract}

\section{Introduction}

The self-inductance of a straight wire may only be defined as so-called partial inductance $[1,2]$. Per se, partial inductance of a single wire cannot be measured. Only loop inductance can be measured. Therefore, partial inductance can only be calculated or determined indirectly from measurements of loop inductance via calculations.

Partial self-inductance of a conductor is defined as the double volume integral of the scalar product of the current density vectors $\overrightarrow{J_{1}}\left(\overrightarrow{r_{1}}\right)$ and $\overrightarrow{J_{2}}\left(\overrightarrow{r_{2}}\right)$ at the points $\overrightarrow{r_{1}}$ and $\overrightarrow{r_{2}}$ divided by the distance $r_{12}$ between these points, carried out over the whole volume of the conductor,

$$
L=\frac{\mu_{0}}{4 \pi} \frac{1}{I^{2}} \iint \overrightarrow{J_{1}} \cdot \overrightarrow{J_{2}} \frac{d \tau_{1} d \tau_{2}}{r_{12}},
$$

where $d \tau_{1}$ and $d \tau_{2}$ are the volume elements around the integration points $\overrightarrow{r_{1}}$ and $\overrightarrow{r_{2}}$, respectively, and where for simplicity we assume non-magnetic conductor material, so that $\mu_{0}$ is the magnetic permeability of the vacuum, $\mu_{0}=4 \pi \cdot 10^{-7} \mathrm{Vs} /(\mathrm{Am})$, and $I$ is the total current flowing in the conductor (see equation (17a), p. 95 in [3]).

In this paper, we present two methods that allow deriving analytic formulae for the partial inductance of straight wires of any cross section and for any frequency. We apply these methods to derive formulae for the cases of circular cross section in the low-frequency limit (where the current distribution is homogeneous) and in the high-frequency limit (where the current is concentrated on the surface of the wire). A couple of formulae for the partial inductance of wires of circular cross section in the low-frequency limit can be found in the literature. We want to compare the accuracies of the various formulae. Since partial inductance cannot be measured, we cannot rely on measurements to assess the analytical results.

But fortunately, for wires of circular cross section in the low-frequency limit it is possible to calculate the partial inductance exactly as the six-dimensional integral of the general inductance definition (1). Instead of taking measurements, we take recourse to such calculations. We present a transformation of coordinates which allows reducing the six-dimensional integral (1) to a threedimensional one. The three-dimensional integral can be computed by means of the function integral 3 which forms part of the MATLAB ${ }^{\circledR}$ programming language.

The analytical formulae which can be found in the literature are all based on the fact that the integration along the wire (i.e. in the direction of current flow) in equation (1) can be carried out in closed form. The integration is done along the longitudinal coordinates $z_{1}$ and $z_{2}$ of the points $\overrightarrow{r_{1}}$ and $\overrightarrow{r_{2}}$ which can independently assume any position along the wire. The result of this two-dimensional integration is the well-known formula for the mutual inductance $M$ of two straight parallel filaments of equal length $l$ separated by a distance $\delta[1-5]$ : 
$M=\frac{\mu_{0}}{2 \pi}\left[l \cdot \log \left(\sqrt{l^{2}+\delta^{2}}+l\right)-l \cdot \log \delta-\sqrt{l^{2}+\delta^{2}}+\delta\right]$,

where $\log$ is the natural logarithm. In order to get the inductance $L$ we need the full six-dimensional integral (1). There remains a four-dimensional integral, namely, the double area integral of the expression (2) over the wire cross section, whereby the points $\overrightarrow{r_{1}}$ and $\overrightarrow{r_{2}}$ can independently assume any position within the cross section, and whereby the normalized current densities $\left|\overrightarrow{J_{1}}\right| / I$ and $\left|\overrightarrow{J_{2}}\right| / I$ of equation (1) must be taken into account. In the low-frequency limit, i.e. for a homogeneous current distribution, the product of the normalized current densities simply reduces to $1 / A^{2}$, where $A$ is the cross sectional area. Thus we need the double area integral of equation (2) normalized by the cross sectional area squared.

The summands of equation (2), except for $-l \cdot \log \delta$, cannot be integrated in closed form. The various formulae for the inductance which can be found in the literature differ in the approximations made in carrying out these integrals and in the number of summands of equation (2) which are considered. Examples of such approximations used in the literature are discussed in section 5. The best variants among them rely on the use of the geometric mean distance (GMD) invented by Maxwell [4] as a solution for the normalized double area integral of $-l \cdot \log \delta$. The result is $-l \cdot \log G M D$, with GMD given by our equation (15). In the literature, rather coarse approximations for the normalized double area integrals of the remaining summands of equation (2) are used, see section 5. We show in sections 810 that in the case of short wires these approximations lead to substantial errors in the calculated inductance.

By contrast, we want to find better approximations which are also valid for short wires. Our first method to derive inductance formulae is the mean distances method described in section 6. It was originally proposed by Rosa [1], although he did not actually carry it out. In this method, in addition to using the GMD as described above, also the arithmetic mean distance (AMD) and the arithmetic mean square distance (AMSD) are used for calculating the normalized double area integrals of the remaining summands of equation (2), see sections 3 and 4. Despite the fact that this method will certainly be more accurate than the coarse ones used in the literature, it is not clear whether it is mathematically well-founded or not. In section 7 we show that it is.

Our second method is the Taylor series method as explained in section 7. It consists of forming the normalized double area integrals by applying the GMD and AMD as in the mean distances method, but expanding the summands $l \cdot \log \left(\sqrt{l^{2}+\delta^{2}}+l\right)$ and $\sqrt{l^{2}+\delta^{2}}$ in equation (2) into a suitable Taylor series, and then replacing $\delta$ with the AMD and $\delta^{2}$ with the AMSD squared.

In section 8 we discuss the errors of the various inductance formulae which were calculated with the help of our exact results. We show that for short wires the AMD and the AMSD may not be neglected anymore. The accuracy of the calculated inductance substantially improves if their exact values are used.
In section 9 we repeat the analysis for the mutual inductance between two identical parallel wires, so that in section 10 we are able to present an application of our theory to the calculation of the inductance of shorted twowire lines. Section 11 closes with our conclusions.

\section{Exact numerical calculation}

For circular cross section the six-dimensional integral (1) can be calculated exactly. If we assume a homogeneous current distribution and denote the radius by $R$, we have $\left|\overrightarrow{J_{1}}\right|=\left|\overrightarrow{J_{2}}\right|=I /\left(\pi R^{2}\right)$, and the integral (1) simplifies to

$$
L=\frac{\mu_{0}}{4 \pi} \frac{1}{\pi^{2} R^{4}} \iint \frac{d \tau_{1} d \tau_{2}}{r_{12}} .
$$

In cylindrical coordinates with radial components $r_{1}$ and $r_{2}$, angular components $\theta_{1}$ and $\theta_{2}$, axial components $z_{1}$ and $z_{2}$, and wire length $l$ the integral reads

$L=\frac{\mu_{0}}{4 \pi^{3} R^{4}} \int_{0}^{l} \int_{0}^{2 \pi} \int_{0}^{R} \int_{0}^{l} \int_{0}^{2 \pi} \int_{0}^{R} \frac{r_{1} r_{2}}{r_{12}} d r_{1} d \theta_{1} d z_{1} d r_{2} d \theta_{2} d z_{2}$.

Let us consider the projections $P_{1}{ }^{\prime}=\left(r_{1}, \theta_{1}\right)$ and $P_{2}{ }^{\prime}=$ $\left(r_{2}, \theta_{2}\right)$ of the integration points $P_{1}=\left(r_{1}, \theta_{1}, z_{1}\right)$ and $P_{2}=\left(r_{2}, \theta_{2}, z_{2}\right)$ onto the $(r, \theta)$-plane as depicted in Fig. 1 . The distance $r_{12}$ ' between the projected integration points $P_{1}{ }^{\prime}$ and $P_{2}{ }^{\prime}$ is then given by the law of cosines. Note that we count $\theta_{1}$ from the ray $\mathrm{OP}_{2}{ }^{\prime}$ to make $r_{12}{ }^{\prime}$ independent of $\theta_{2}$.

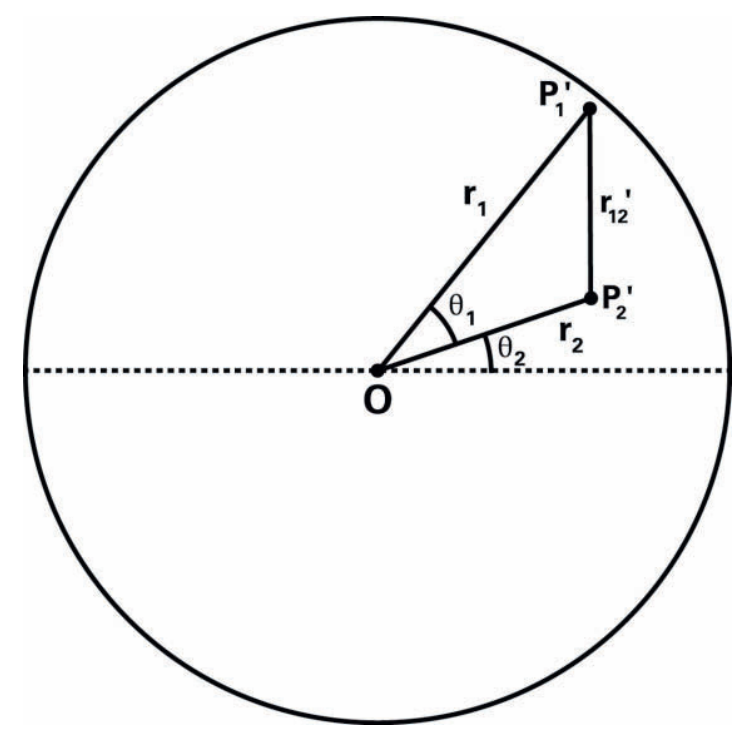

Figure 1: Polar coordinates $\left(r_{1}, \theta_{1}\right)$ and $\left(r_{2}, \theta_{2}\right)$ of the projected integration points $P_{1}{ }^{\prime}$ and $P_{2}{ }^{\prime}$ with their projected distance $r_{12}{ }^{\prime}$.

Thus, the full distance $r_{12}$ (including the axial components $z_{1}$ and $z_{2}$ ) can be written as

$$
r_{12}=\sqrt{r_{1}^{2}+r_{2}^{2}-2 r_{1} r_{2} \cos \theta_{1}+\left(z_{1}-z_{2}\right)^{2}},
$$

and the integral (4) reduces to 


$$
L=\frac{\mu_{0}}{2 \pi^{2} R^{4}} \int_{0}^{l} \int_{0}^{R} \int_{0}^{l} \int_{0}^{2 \pi} \int_{0}^{R} \frac{r_{1} r_{2}}{r_{12}} d r_{1} d \theta_{1} d z_{1} d r_{2} d z_{2} .
$$

The double volume integral (1) is well-defined (see $\S 15 \mathrm{D}$, p. 98 in [3]). But in the form (6) it is not suitable for numerical integration because for $z_{1}=z_{2}, r_{1}=r_{2}, \theta_{1}=0$, the integrand has a pole singularity.

The singularity can be avoided by using cylinder coordinates for $P_{1}$ with axis through $P_{2}$, as shown in projection in Fig. 2. The projected point $P_{1}{ }^{\prime}$ now has polar coordinates $P_{1}^{\prime}=(s, \psi)$, where $s=r_{12}{ }^{\prime}$, and $P_{1}$ has cylinder coordinates $P_{1}=\left(s, \psi, z_{1}\right)$. For the inner integral in equation (3) we can use $d \tau_{1}=s^{\cdot} d \psi d s d z_{1}$. The integral covers two regions which are separated by the dotted circle in Fig. 2. Its radius is $R-r_{2}$.

Within the dotted circle, $\mathrm{s}$ runs from 0 to $R-r_{2}$. Since the limits of the angle $\psi$ outside the dotted circle are symmetrical (see Fig. 2), we also use symmetrical limits within the circle, i.e. $-\pi$ and $\pi$.

Outside the dotted circle, $s$ runs from $R-r_{2}$ to $R+r_{2}$. In this region the limit $\Psi$ of the angle $\psi$ depends on $s$ and can be computed from the law of cosines (see Fig. 2),

$$
R^{2}=r_{2}^{2}+s^{2}-2 r_{2} s \cdot \cos \Psi .
$$

This yields

$$
\Psi=\Psi\left(s, r_{2}\right)=\left\{\begin{array}{cl}
\arccos \frac{r_{2}{ }^{2}-R^{2}+s^{2}}{2 r_{2} s}, & s \geq R-r_{2} \\
\pi, & s \leq R-r_{2}
\end{array}\right.
$$

Using $r_{12}=\sqrt{s^{2}+\left(z_{1}-z_{2}\right)^{2}}$ the integral (3) becomes $L=\frac{\mu_{0}}{4 \pi^{3} R^{4}} \int_{0}^{l} \int_{0}^{R} \int_{0}^{2 \pi} \int_{0}^{R+r_{2}} \int_{0}^{l} \int_{-\Psi}^{\Psi} \frac{s \cdot r_{2}}{\sqrt{s^{2}+\left(z_{1}-z_{2}\right)^{2}}} d \psi d z_{1} d s d \theta_{2} d r_{2} d z_{2}$.

Now the integrations over $\psi$ and $\theta_{2}$ can be carried out, since the integrand is independent of these variables. They simply yield $4 \pi \Psi\left(\mathrm{s}, \mathrm{r}_{2}\right)$, and the integral (9) reduces to

$$
L=\frac{\mu_{0}}{\pi^{2} R^{4}} \int_{0}^{l} \int_{0}^{R} \int_{0}^{R+r_{2}} \int_{0}^{l} \frac{\Psi\left(\mathrm{s}, r_{2}\right) \cdot s \cdot r_{2}}{\sqrt{s^{2}+\left(z_{1}-z_{2}\right)^{2}}} d z_{1} d s d r_{2} d z_{2} .
$$

Further, the integration over $z_{1}$ can be carried out with the help of the substitution

$$
t=z_{1}-z_{2}, d z_{1}=d t
$$

and integral no. 241 (first equation), p. 313 in [6]. With $a=1, b=0$ and $c=s^{2}$ in the notation of [6], we have in our notation

$$
\begin{aligned}
& \Lambda(s)=\Lambda\left(s, z_{2}\right):=\int_{0}^{l} \frac{d z_{1}}{\sqrt{s^{2}+\left(z_{1}-z_{2}\right)^{2}}}= \\
& =\log \frac{\left(z_{2}+\sqrt{z_{2}^{2}+s^{2}}\right)\left(l-z_{2}+\sqrt{\left(l-z_{2}\right)^{2}+s^{2}}\right)}{s^{2}} .
\end{aligned}
$$

So we have reduced the six-dimensional integral (3) to a three-dimensional one:

$$
L=\frac{\mu_{0}}{\pi^{2} R^{4}} \int_{0}^{l} \int_{0}^{R} \int_{0}^{R+r_{2}} \Psi\left(s, r_{2}\right) \Lambda\left(s, z_{2}\right) s r_{2} d s d r_{2} d z_{2} .
$$

In this form the integral is not yet suitable for numerical integration because the integrand is not differentiable at $s=R-r_{2}$. In fact, the one-sided derivatives of $\Psi(s)$ at $s=R-r_{2}$ are 0 and $-\infty$. Breaking the integral over $s$ up at this point, we finally get

$$
\begin{aligned}
L= & \frac{\mu_{0}}{\pi^{2} R^{4}}\left[\pi \int_{0}^{l} \int_{0}^{R} \int_{0}^{R-r_{2}} \Lambda\left(s, z_{2}\right) s r_{2} d s d r_{2} d z_{2}+\right. \\
& \left.+\int_{0}^{l} \int_{0}^{R} \int_{R-r_{2}}^{R+r_{2}} \Psi\left(s, r_{2}\right) \Lambda\left(s, z_{2}\right) s r_{2} d s d r_{2} d z_{2}\right]
\end{aligned}
$$

Further, the integrand must be set to 0 for $s<\varepsilon$ for some sufficiently small $\varepsilon$, because the function $\Lambda\left(s, z_{2}\right)$ has a logarithmic singularity at $s=0$. In our implementation we use twice the relative machine tolerance for $\varepsilon$ (which is less than $10^{-15}$ ).

The singularity problem of our original integral (3) or (6) is thereby solved, since the remaining factors of the integrands of equation (14) are differentiable in the interior of the domain of integration. The integrals (14) can then easily be computed with the help of the MATLAB ${ }^{\circledR}$ function integral3.

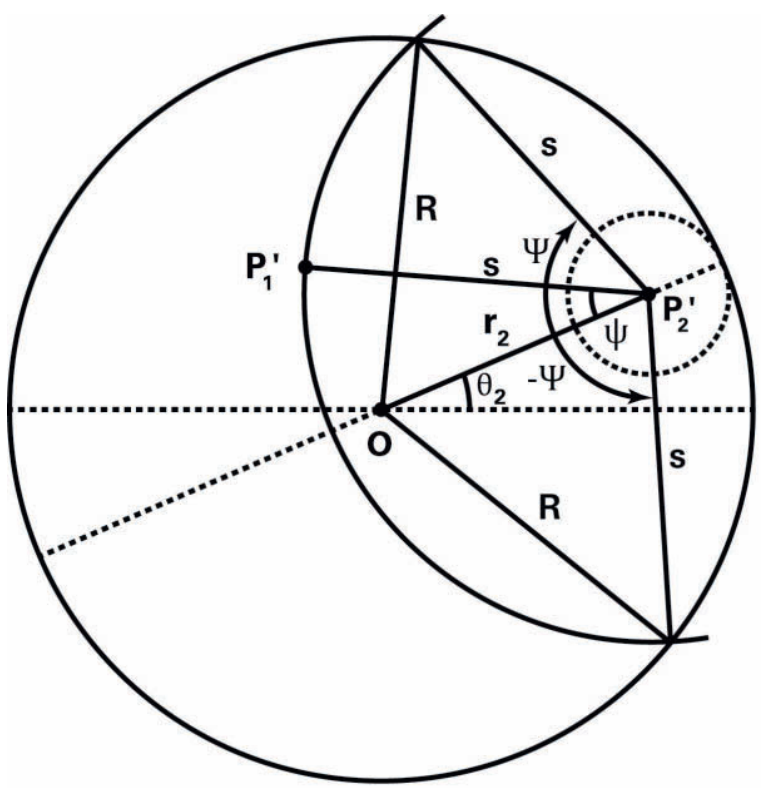

Figure 2: Polar coordinates $(s, \psi)$ for the projected integration point $P_{1}{ }^{\prime}$. For constant $s$, the angle $\psi$ varies between $-\Psi$ and $+\Psi$.

\section{Calculation of the mean distances}

As we have already explained in the introduction, the calculation of the normalized double area integral of the summand $-l \cdot \log \delta$ in equation (2) over the cross section of the wire amounts to calculating the logarithm of the geometric mean distance (GMD) of the cross section. Its logarithm is the mean value of the logarithm of the distance $\delta$ between two arbitrary points within the cross section, which is a disk of radius $R$. Thus, the integral or mean is simply $-l \cdot \log G M D$. For disks of radius $R$, the GMD was given by Maxwell (see $\S 692$ (9), p. 328 in [4]). It is

$$
G M D=R \cdot \mathrm{e}^{-\frac{1}{4}}=0.7788008 \cdot R .
$$


The arithmetic mean square distance (AMSD) squared is, analogously, the normalized double area integral or mean of $\delta^{2}$ over the cross section. For disks of radius $R$, we have, by definition,

$$
A M S D^{2}=\frac{1}{\pi^{2} R^{4}} \int_{0}^{2 \pi} \int_{0}^{2 \pi} \int_{0}^{R} \int_{0}^{R} r_{12}{ }^{2} r_{1} r_{2} d r_{1} d r_{2} d \theta_{1} d \theta_{2},
$$

where $\delta^{2}$ is now written as $r_{12}{ }^{2}$ and is the square of the distance between the two integration points, which is given by the law of cosines as (see Fig. 1)

$$
r_{12}^{2}=r_{1}^{2}+r_{2}^{2}-2 r_{1} r_{2} \cos \theta_{1}
$$

The integral (16) can be carried out in closed form. The result is simply

$$
A M S D=R .
$$

The arithmetic mean distance (AMD) is the normalized double area integral of $\delta$ over the cross section. It is calculated analogously to equation (16), except that the integrand is the distance $r_{12}$ between the two integration points, rather than its square. Since the integrand does not depend on $\theta_{2}$, the integral reduces to

$$
A M D=\frac{2}{\pi R^{4}} \int_{0}^{2 \pi} \int_{0}^{R} \int_{0}^{R} r_{12} r_{1} r_{2} d r_{1} d r_{2} d \theta_{1} .
$$

This integral cannot be solved analytically. Clearly, the AMD must be proportional to $R$, so that the problem reduces to determining the proportionality factor, which is defined by the above integral for $R=1$.

With the help of the MATLAB $\AA$ function integral3 the integral can be evaluated numerically by writing two lines of code (lines end with a semicolon):

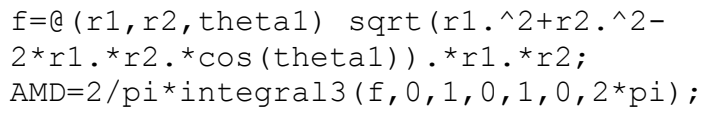

The final result is

$$
A M D=0.905415 \cdot R \text {. }
$$

Incidentally, if one replaces sqrt by $1 / 2 * \log$ in the first line of code above, one gets 0.7788008 , the proportionality factor in the formula for the GMD, see equation (15).

\section{Mean distances in the high-frequency limit}

In the high-frequency limit the current is concentrated on the boundary of the wire. In the case of circular cross section the mean distances then reduce to those of a circle. The corresponding two-dimensional integrals can all be solved in closed form. The GMD of a circle of radius $R$ is simply (see $\S 692$ (9), p. 328 in [4])

$$
G M D_{H F}=R \text {. }
$$

The AMSD squared for high frequencies is, by definition,

$$
A M S D_{H F}^{2}=\frac{1}{4 \pi^{2}} \int_{0}^{2 \pi} \int_{0}^{2 \pi} r_{12}^{2} d \theta_{1} d \theta_{2} .
$$

Here we have $r_{1}=r_{2}=R$, hence, by equation (17),

$$
r_{12}^{2}=2 R^{2}\left(1-\cos \theta_{1}\right) .
$$

Insertion into equation (22) yields

$$
A M S D_{H F}=\sqrt{2} R \text {. }
$$

The AMD for high frequencies is defined analogously as

$$
A M D_{H F}=\frac{1}{4 \pi^{2}} \int_{0}^{2 \pi} \int_{0}^{2 \pi} r_{12} d \theta_{1} d \theta_{2} .
$$

The result is

$$
A M D_{H F}=\frac{4}{\pi} R .
$$

For intermediate frequencies, equation (1) must be used instead of equation (3). In the integrals for the mean distances then appear weighting factors that correspond to the normalized current densities $\left|\overrightarrow{J_{1}}\right| / I$ and $\left|\overrightarrow{J_{2}}\right| / I$ at the particular frequency.

\section{Inductance formulae from the literature}

Various analytical formulae for the inductance of rectangular coils of circular cross section can be found in the literature. They are all based on equations (1) and (2), at least implicitly, and on the assumption of a homogeneous current distribution. But they differ in the way equation (2) is integrated over the cross section. Usually, only the composed formula for the inductance of a complete rectangular coil is given, and not the corresponding formula for the partial inductance of a single wire. But since all these formulae are based on a formula for a single wire, it is not difficult to re-derive the underlying single wire formula, which, in this paper, is what we are interested in. For easier comparison we present all inductance formulae in the same expanded form.

The simplest approach is to take equation (2) without integrating it at all, as adopted by King and Prasad (see equation (9.5-21), p. 345 in [7]), simply replacing $\delta$ by $R$ and neglecting $R^{2}$ against $l^{2}$ in the square roots. This amounts to taking the high-frequency limit for the GMD instead of the low-frequency limit, see equations (15) and (21), to neglecting the AMSD altogether, and to adopting the value $R$ for the AMD, which is too large for the lowfrequency limit, see equation (20), and too low for the highfrequency limit, see equation (26).

Unfortunately, King and Prasad don't mention for what frequencies their formula is supposed to be valid. Their formula for the loop inductance of a rectangular coil of length $a$, width $b$, diagonal of the rectangle $D=\sqrt{a^{2}+b^{2}}$, and radius $R$ is

$$
L_{\text {coil }}=\frac{\mu_{0}}{\pi}\left[a \cdot \log \frac{2 a b}{R(a+D)}+b \cdot \log \frac{2 a b}{R(b+D)}+2(D-a-b+R)\right] .
$$

The corresponding single wire formula from which their coil formula (27) is derived can thus be re-derived as

$$
L=\frac{\mu_{0}}{2 \pi}[l \cdot \log (2 l)-l \cdot \log R-l+R] .
$$

Meinke and Gundlach (see p. E14 in [8]) present a similar formula, but they use 0.80 for the proportionality factor in 
the formula for the GMD instead of 0.7788008 , and they neglect both the AMSD and the AMD:

$$
L=\frac{\mu_{0}}{2 \pi}\left[l \cdot \log (2 l)-l \cdot \log \left(\frac{4}{5} R\right)-l\right] .
$$

Wien (see p. 939 in [9]) uses the exact value for the GMD, but he also neglects both the AMD and the AMSD:

$$
L=\frac{\mu_{0}}{2 \pi}\left[l \cdot \log (2 l)-l \cdot\left(\log R-\frac{1}{4}\right)-l\right] .
$$

Rosa (see equation (24), p. 319 in [1]) also takes the exact value for the GMD and neglects the AMSD, but he does take the AMD into account, although he simply equals it to $R$, as King and Prasad did, which is too large. Rosa's result can be written in the form

$$
L=\frac{\mu_{0}}{2 \pi}\left[l \cdot \log (2 l)-l \cdot\left(\log R-\frac{1}{4}\right)-l+R\right] .
$$

Paul calculates the internal and external inductivity of a round wire separately via the magnetic fields (see equations (4.80), p. 164 and (5.18a), p. 207 in [2]). He also approximates the AMD by $R$. Apart from this approximation, the formula combined from Paul's equations is identical to our result (34) below. The combined formula is

$$
L=\frac{\mu_{0}}{2 \pi}\left[l \cdot \log \left(\sqrt{l^{2}+R^{2}}+l\right)-l \cdot\left(\log R-\frac{1}{4}\right)-\sqrt{l^{2}+R^{2}}+R\right] .
$$

This formula does not appear in Paul's book, though, nor does a formula for the self-induction of a loop coil that is derived from it.

\section{The mean distances method}

In the mean distances method we use the mean distances in order to compute the normalized double area integrals or means of the summands of equation (2) over the cross section of the wire. Thus, as described in section 3 , the mean of $-l \cdot \log \delta$ is $-l \cdot \log G M D$, and the mean of $\delta$ is just the AMD.

For a disk the AMD in the low-frequency limit must be computed numerically, see equation (20). In the highfrequency limit it can be calculated analytically, see equation (26).

Finally, the means of the remaining summands in equation (2), i.e., $\quad l \cdot \log \left(\sqrt{l^{2}+\delta^{2}}+l\right)$ and $\sqrt{l^{2}+\delta^{2}}$, are approximated by replacing $\delta^{2}$ with $A M S D^{2}$. But replacing $\delta^{2}$ by $A M S D^{2}$ is clearly not the same as calculating the mean of $\log \left(\sqrt{l^{2}+\delta^{2}}+l\right)$ and $\sqrt{l^{2}+\delta^{2}}$ over the cross section. Thus, it is not clear whether this method is mathematically well-founded, even if it is evident that it must be more accurate than the coarse approximations made in the formulae from the literature. In the next section we prove that the method is, indeed, well-founded.

Carrying out all the mentioned replacements of $\delta$ in equation (2), we get the general result

$$
\begin{gathered}
L=\frac{\mu_{0}}{2 \pi}\left[l \cdot \log \left(\sqrt{l^{2}+A M S D^{2}}+l\right)-l \cdot \log G M D\right. \\
\left.-\sqrt{l^{2}+A M S D^{2}}+A M D\right] .
\end{gathered}
$$

This result is general in the sense that it applies to wires of any cross section, not just circular, and for any frequency. Moreover, if the integrals for the mean distances are not extended twice over the same cross section, but over two different cross sections of two parallel wires, then one gets the GMD, AMSD, and AMD for two parallel wires. Thus, equation (33) is also valid for the mutual inductance $M$ of two parallel wires of equal length. We make use of this fact in section 9 .

Using the specific values for GMD, AMSD and AMD as given by equations (15), (18) and (20), we get the result in a form which can directly be compared with the lowfrequency formulae from the literature given in equations (28) to (32):

$$
\begin{gathered}
L=\frac{\mu_{0}}{2 \pi}\left[l \cdot \log \left(\sqrt{l^{2}+R^{2}}+l\right)-l \cdot\left(\log R-\frac{1}{4}\right)\right. \\
\left.-\sqrt{l^{2}+R^{2}}+0.905415 \cdot R\right] .
\end{gathered}
$$

In the high-frequency limit we use the specific values for the mean distances given by equations (21), (24) and (26) with the result

$$
L_{H F}=\frac{\mu_{0}}{2 \pi}\left[l \cdot \log \left(\sqrt{l^{2}+2 R^{2}}+l\right)-l \cdot \log R-\sqrt{l^{2}+2 R^{2}}+\frac{4}{\pi} R\right] .
$$

\section{The Taylor series method}

An even more precise approximation to the exact integral (3) can be obtained by using the GMD and AMD for the corresponding means, as in the mean distances method, but using Taylor series expansions in

$$
x=\frac{\delta}{l}
$$

in the summands $l \cdot \log \left(\sqrt{l^{2}+\delta^{2}}+l\right)$ and $\sqrt{l^{2}+\delta^{2}}$ of equation (2) about a suitable point $x_{0}$.

For the Taylor series of the square root $\sqrt{l^{2}+\delta^{2}}$ we find, up to second order of expansion,

$$
\begin{aligned}
& \sqrt{l^{2}+\delta^{2}}=l \cdot \sqrt{1+x^{2}} \approx \\
& \approx l \cdot \omega+\frac{l x_{0}}{\omega}\left(x-x_{0}\right)+\frac{l}{2 \omega^{3}}\left(x-x_{0}\right)^{2},
\end{aligned}
$$

where

$$
\omega=\sqrt{1+x_{0}^{2}} \text {. }
$$

We use the expansion point corresponding to $\delta=A M S D$ (see equation (36)), i.e.

$$
x_{0}=\frac{A M S D}{l} .
$$

This minimizes the maximum of $\left|x-x_{0}\right|$ for a disk because we then have $A M S D=R$, equation (18). For a general cross 
section the corresponding minimizer is $x_{0}=D /(2 l)$ where $D$ is the diameter of the cross section. With the abbreviation

$$
\Delta:=A M S D-A M D>0
$$

we find for the means of the differences $\left(x-x_{0}\right)$ and $\left(x-x_{0}\right)^{2}$ in the Taylor series (37)

$$
\operatorname{mean}\left(x-x_{0}\right)=-\frac{\Delta}{l}
$$

and

$$
\operatorname{mean}\left(\left(x-x_{0}\right)^{2}\right)=2 A M S D \cdot \frac{\Delta}{l^{2}} .
$$

Note that $\Delta$ is always positive for any cross section. This follows from the Cauchy-Schwarz inequality.

The mean of the square root over the cross section is then

$$
\operatorname{mean}\left(\sqrt{l^{2}+\delta^{2}}\right) \approx W-\frac{A M S D}{W} \Delta+\frac{l^{2}}{W^{3}} A M S D \cdot \Delta,
$$

where

$$
W:=\sqrt{l^{2}+A M S D^{2}}=l \cdot \omega .
$$

For the Taylor expansion of the logarithmic expression, we find analogously

$$
\begin{gathered}
l \cdot \log \left(\sqrt{l^{2}+\delta^{2}}+l\right)=l \cdot\left[\log l+\log \left(\sqrt{1+x^{2}}+1\right)\right] \\
\approx l \cdot \log l+l \cdot \log (\omega+1)+\frac{l x_{0}}{\omega(\omega+1)}\left(x-x_{0}\right) \\
+\frac{\left.l\left(\omega-x_{0}\right)^{2}\right)}{2 \omega^{3}(\omega+1)}\left(x-x_{0}\right)^{2}
\end{gathered}
$$

and for its mean over the cross section we get

$$
\begin{aligned}
& \text { mean }\left[l \cdot \log \left(\sqrt{l^{2}+\delta^{2}}+l\right)\right] \\
& \approx l \cdot \log (W+l)-\frac{l \cdot A M S D}{W(W+l)} \Delta+\frac{l\left(l \cdot W-A M S D^{2}\right)}{W^{3}(W+l)} A M S D \cdot \Delta,
\end{aligned}
$$

where, again, $\omega$ and $W$ are given by equations (38) and (44), we have expanded the series about the point $x_{0}$ defined in equation (39), and the means of the differences $\left(x-x_{0}\right)$ and $\left(x-x_{0}\right)^{2}$ in the Taylor series (45) are given by equations (41) and (42), respectively.

By definition (44), the constant term $W$ in the series (43) is identical to the square root term $\sqrt{l^{2}+A M S D^{2}}$ in equation (33). Likewise, the constant term $l \cdot \log (W+l)$ in the series (46) is identical to the corresponding logarithmic term $l \cdot \log \left(\sqrt{l^{2}+A M S D^{2}}+l\right)$ in equation (33). Since the remaining terms in equation (33) are exact, equations (43) and (46) show that the inductance formula based on the mean distances method is the Taylor expansion of order zero.

This theoretical result proves that the formula obtained with the mean distances method represents an approximation converging to the exact solution for $x$ close to $x_{0}$, i.e. for those cross sections for which the AMD does not deviate too much from the AMSD. This proves that the mean distances method is mathematically well-founded. For long wires, its formulae converge to the exact solution. It also shows that the rate of convergence depends on the geometrical shape of the cross section.
For disks the AMD deviates by only $-9.5 \%$ from the AMSD (as follows from equations (18) and (51)). This means that the condition $A M D \approx A M S D$ is met for circular cross section. In the next section we show that the mean distances method yields very good results, even for very short wires. The additional four terms in equations (43) and (46) represent corrections for the deviation of the AMD from the AMSD. These equations allow us to derive a general expression for the correction $\Delta L$ which must be added to the inductance $L$ given by the general inductance formula (33):

$$
\Delta L=\frac{\mu_{0}}{2 \pi} A M S D \cdot \Delta\left[\frac{1}{W}-\frac{l^{2}}{W^{3}}-\frac{l}{W(W+l)}+\frac{l\left(l \cdot W-A M S D^{2}\right)}{W^{3}(W+l)}\right] .
$$

(Note that the square root term $\sqrt{l^{2}+\delta^{2}}$ in equation (2) has negative sign). Expanding the fractions in equation (47) to the same denominator, collecting terms and applying definition (44) we get the final result

$$
\Delta L=\frac{\mu_{0}}{2 \pi} A M S D \cdot \Delta \frac{W-l}{W(W+l)} .
$$

In the same way as equation (33), equation (48), with $W$ according to the general definition (44), is also valid for the correction $\Delta M$ to the mutual inductance $M$ of parallel wires of equal length. We apply equation (48) to $\Delta M$ in section 9 .

For circular cross sections and low frequencies we set $A M S D=R$ (equation (18)) and therefore

$$
\begin{aligned}
& W=\sqrt{l^{2}+R^{2}}=: \Omega, \\
& \Delta=R-A M D=k R, \\
& k:=1-\frac{A M D}{R}=0.094585 .
\end{aligned}
$$

We then get the following expression for the correction for wires of circular cross section and low frequencies:

$$
\Delta L=\frac{\mu_{0}}{2 \pi} k R^{2} \frac{\Omega-l}{\Omega(\Omega+l)} .
$$

\section{Results and discussion for the partial self- inductance}

In the case of homogeneous current distribution over the cross section, the design of a single wire may be described by just one dimensionless parameter (as far as the accuracy of inductance calculations is concerned): the ratio $l / R$ of wire length to radius. When this ratio is large, then even the simplest formula will do. But for shorter wires, a more accurate formula is needed.

Figures 3-7 show the relative error of the various inductance formulae presented in section 5 . The error is plotted as a function of the ratio $l / R$ ranging from 2 to 20. Exact values as calculated in section 2 were used as reference values to compute the error. The corresponding exact inductance values for a wire of radius $1 \mathrm{~mm}$ range from $0.41 \mathrm{nH}$ to 11.9 $\mathrm{nH}$

Fig. 3 shows the relative errors of formula (28) from King and Prasad [7]. In this figure only, the plot starts with the ratio 1. For large ratios the formula converges only very 
slowly to the exact value. The reason is that the value for the GMD used corresponds to the high-frequency limit. The AMSD is neglected. It is interesting to note that at the ratio $l / R=2.5$ the curve reaches its minimum value $-14.7 \%$. For lower ratios it increases to zero and then changes sign. For still lower ratios, it finally increases without bound. The reason for the existence of a minimum is that for small ratios $l / R$, the calculated inductance becomes very sensitive on the particular value of the AMD used. When it is too small or even neglected, then the error is negative, and for small ratios it decreases without bound as in Fig. 4. When it is only slightly too large as in equation (28) depicted in Fig. 3 , then the error increases without bounds. The combined effect of the wrong value for the GMD and of the slightly too large value of the AMD leads to the minimum observed in Fig. 3.

Fig. 4 shows the error of formulae (29) and (30) stemming from Meinke-Gundlach [8] and Wien [9], respectively. For large ratios these formulae converge considerably faster to the exact value than formula (28) in Fig. 3. This is due to the more accurate values for the GMD used in these formulae. Wien's formula converges faster with an error of $-1.5 \%$ at the ratio 20 , compared to $-2.4 \%$ for Meinke and Gundlach. This is because Wien uses the exact value for the GMD, namely $0.7788008 \cdot R$, whereas Meinke and Gundlach use $0.8 \cdot R$. For lower ratios both equations produce large negative errors whose magnitude is larger than the one of equation (28) plotted in Fig. 3. The reason is that in equations (29) and (30) the AMD is completely neglected, in contrast to equation (28). The neglect of the AMD is also the reason why the error of these equations is negative and why for small ratios it decreases without bound.

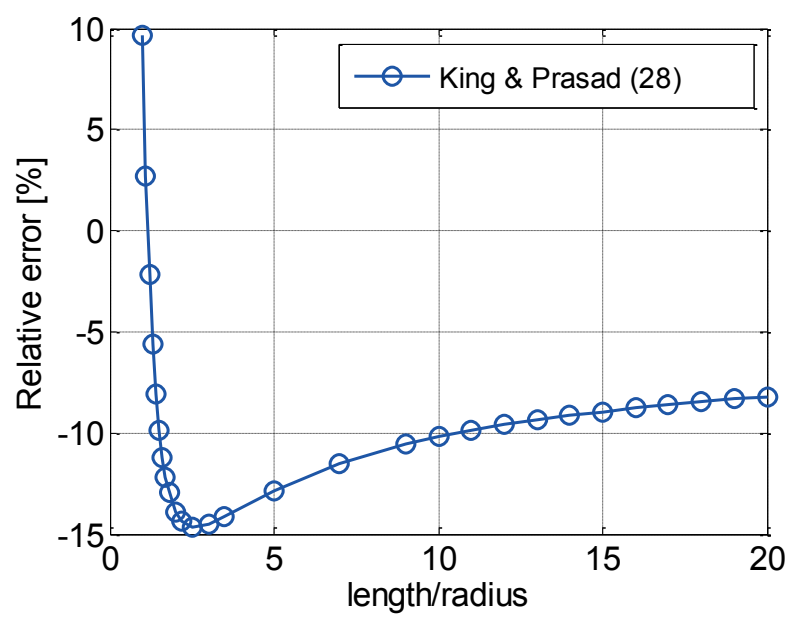

Figure 3: Relative error of formula (28) due to King and Prasad, plotted as a function of the ratio of length to radius.

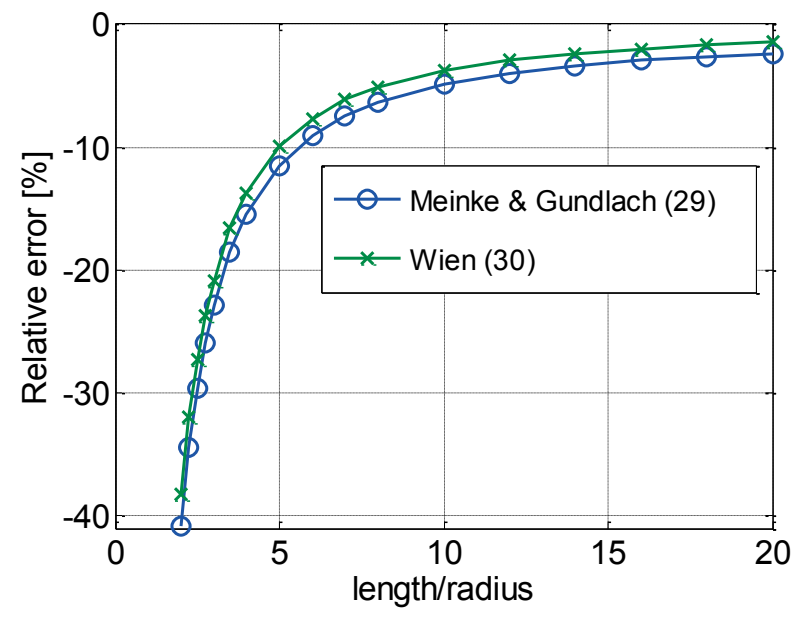

Figure 4: Relative errors of formulae (29) and (30) due to Meinke-Gundlach and Wien, respectively.

Fig. 5 depicts the errors of formulae (31) and (32) due to Rosa [1] and Paul [2], respectively (although formula (32) does not actually appear in [2]). The errors are now positive. The only difference between the curves of Fig. 4 and Fig. 5 is that in the formulae of Fig. 5 the AMD is taken into account, albeit in both curves with a value which is slightly too large $(R$ instead of $0.905415 \cdot R)$, whereas in those of Fig. 4 it is neglected altogether. The magnitude of the error is 4 to 15 times smaller in Fig. 5 than in Fig. 4. This shows that the AMD may not be neglected if accurate results are needed. The difference between Rosa's and Paul's equation is that Rosa neglects the AMSD, whereas Paul uses its exact value $A M S D=R$. It comes as no surprise that Rosa's neglect of the AMSD, which means neglecting $R^{2}$ against $l^{2}$ in the square roots of equation (2), is no longer valid for small ratios $l / R$.

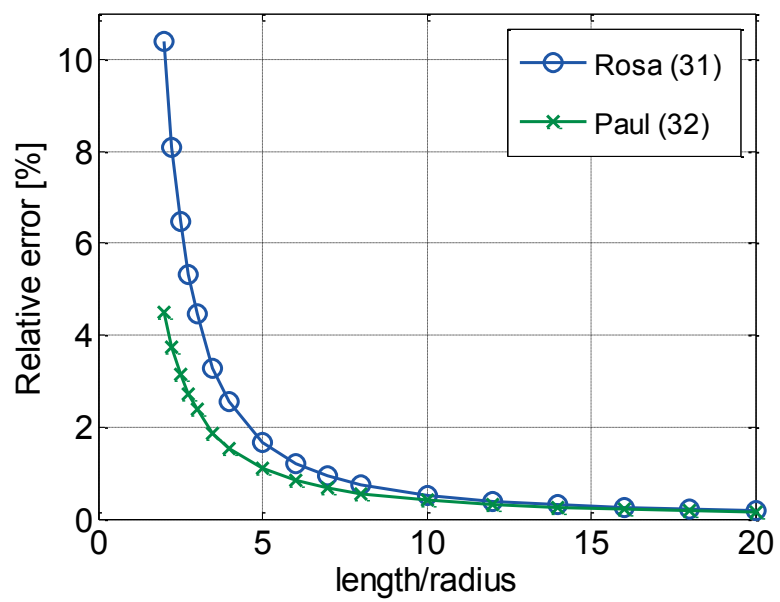

Figure 5: Relative errors of formulae (31) and (32) due to Rosa and Paul, respectively. 


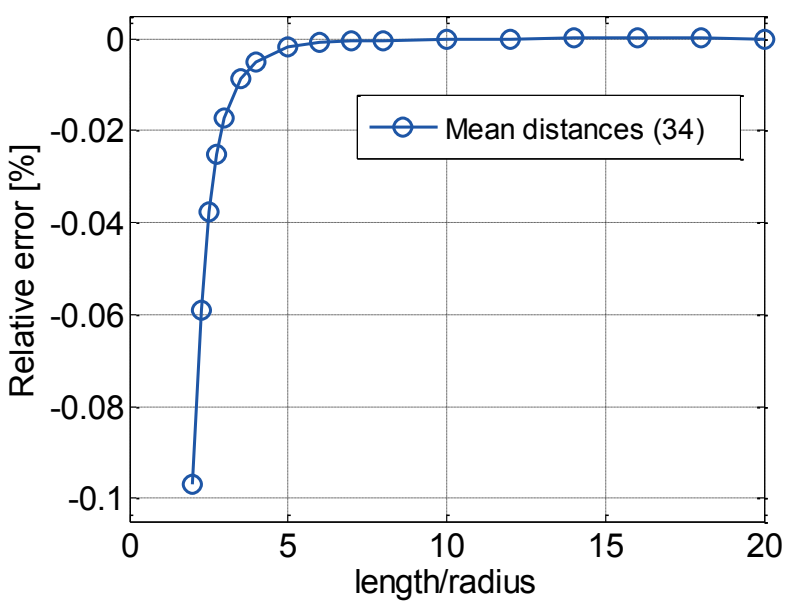

Figure 6: Relative error of formula (34) derived with the mean distances method.

The importance of the particular value of the AMD used is evidenced by Fig. 6 which shows the error of equation (34) derived with the mean distances method. The only difference of this formula to Paul's is the use of the exact value of the AMD. The improvement in accuracy brought about by this minor change is dramatic. For the lowest ratio 2 the accuracy improves by a factor of 104 compared to Rosa's and a factor of 46 compared Paul's formula. For the largest ratio 20 the improvement factor increases to 700 compared to both Rosa and Paul. It is quite remarkable that such a simple formula (34), which is barely more complicated than the formulae from the literature, produces so much better results. This again testifies to the importance of using the exact values for both the AMSD and the AMD in inductance calculations for short wires.

Finally, Fig. 7 shows the relative error of the Taylor series method. It consists of adding to the mean distances formula (34) the correction term given by equation (52). The error is now so small that for practical purposes, this method might even be used in place of the exact calculation.

Our results show that for short wires, the AMSD and the AMD cannot be neglected anymore. Furthermore, they demonstrate that for very short wires it is not sufficient to simply approximate the AMD by $R$, but that the exact value according to equation (20) must be used.

\section{Mutual inductance calculations}

In applications of the theory to conducting structures composed of two or more wires, like two-wire lines or loop coils, we also need precise formulae for the mutual inductance of parallel wires. In this section, we derive such formulae for round wires and compare them to the formula known from the literature, where again our reference values are exact values that are computed numerically.

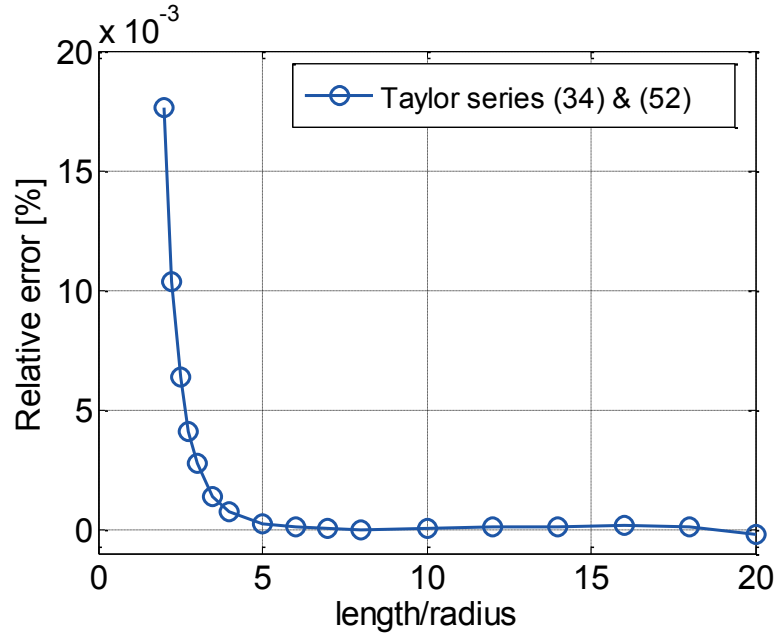

Figure 7: Relative error of formulae (34) and (52) derived with the Taylor series method.

Our general equations (33) and (48) are also valid for the mutual inductance $M$ of two parallel wires of equal length. And in the same way as we re-derived the formulae for the partial inductance of single wires of circular cross section from the formulae for the self-inductance of loop coils found in the literature, we can also re-derive the corresponding formulae for the mutual inductance of parallel wires. All authors of the formulae we discuss in this paper used the same expressions for the means of all the summands in equation (2). They are simply obtained from equation (2) by replacing $\delta$ with $d$, the distance between the centers of the wires:

$$
M=\frac{\mu_{0}}{2 \pi}\left[l \cdot \log \left(\sqrt{l^{2}+d^{2}}+l\right)-l \cdot \log d-\sqrt{l^{2}+d^{2}}+d\right] .
$$

Equation (53) corresponds to using the exact value for the GMD because for two disjoint disks whose centers are separated by a distance $d$, the GMD is just $d$ (this follows from $\S 692$ (7), p. 328 in [4]). A modern proof uses twice the mean value property of harmonic functions, applied to the function $\log r$, which is harmonic in the plane (except at the origin). In order to distinguish this GMD from the single wire value, we use the designation $G M D_{d}$ for the geometric mean distance of two wires separated by a distance $d$ :

$$
G M D_{d}=d .
$$

Further, the procedure leading to equation (53) corresponds to approximating both the AMSD and the AMD by $d$.

For the exact calculation of the AMSD of two disjoint disks whose centers are separated by a distance $d$, see Fig. 8. $P_{1}$ is an arbitrary point within the first disk and $P_{2}$ within the second. As in Fig. 1 we make use of the freedom to define the angle $\theta_{1}$ of the polar coordinates, see Fig. 8. By virtue of the law of cosines, we have

$$
\begin{aligned}
& r_{02}^{2}=d^{2}+r_{2}^{2}-2 d \cdot r_{2} \cos \theta_{2}, \\
& r_{12}^{2}=r_{1}{ }^{2}+r_{02}{ }^{2}-2 r_{1} r_{02} \cos \theta_{1} .
\end{aligned}
$$

Inserting equation (55) into equation (56) yields 


$$
\begin{aligned}
r_{12}{ }^{2}= & r_{1}{ }^{2}+d^{2}+r_{2}{ }^{2}-2 d \cdot r_{2} \cos \theta_{2}- \\
& 2 r_{1} \sqrt{d^{2}+r_{2}{ }^{2}-2 d \cdot \cos \theta_{2}} \cos \theta_{1} .
\end{aligned}
$$

The AMSD squared is then, by definition, given by equation (16) with the distance $r_{12}$ between the points $P_{1}$ and $P_{2}$ taken from equation (57). The result for two disks of radius $R$ is

$$
A M S D_{d}^{2}=d^{2}+R^{2}
$$

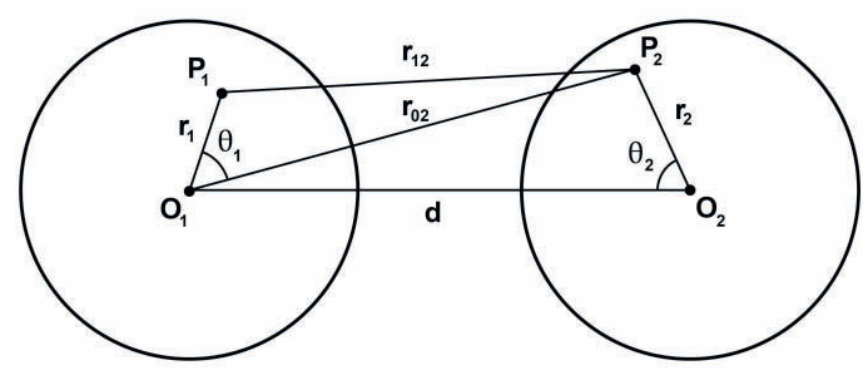

Figure 8: Polar coordinates $\left(r_{1}, \theta_{1}\right)$ and $\left(r_{2}, \theta_{2}\right)$ for the point $P_{1}$ in the cross section of the first wire and $P_{2}$ in that of the second.

As was the case for a single disk in section 3, the AMD for two disks, i.e. integral (16) with $r_{12}^{2}$ replaced by $r_{12}$ by taking the square root of equation (57), cannot be obtained in closed form. By iterating the function integral2 for the computation of two-dimensional integrals in MATLAB $\AA$, the four-dimensional integral for the AMD can be calculated numerically. We have found that for two disks of radius $R$ it can be approximated with good accuracy by the expression

$$
A M D_{d} \approx d+\frac{R^{2}}{4 d}
$$

In normalized form, this can be written as

$$
A M D_{d} \approx R\left(\kappa+\frac{1}{4 \kappa}\right)=d\left(1+\frac{1}{4 \kappa^{2}}\right)
$$

with the parameter

$$
\kappa=\frac{d}{R} .
$$

For $\kappa=1$ the approximated value for the AMD is $2.7 \%$ too low, for $\kappa=2$ it is $0.18 \%$, for $\kappa=3$ it is $0.03 \%$, and for $\kappa=10$ it is $2.6 \cdot 10^{-4} \%$. Note that the AMD is only usefully defined for $\kappa \geq 2$ because otherwise the two wires would overlap. With the value $\kappa=2$ they touch. But since this value does not lead to a singularity in the AMD, it may be used as the limiting value for an infinitely thin insulating gap between the wires.

There are two dimensionless parameters to describe an arrangement of two identical parallel wires (as far as the accuracy of the calculation of their mutual inductance is concerned): the ratios $l / R$ and $\kappa=d / R$. We have only one formula from the literature, namely, equation (53). Note that it is independent of $R$ (it assumes $R \approx 0$ ).

In order to find the formula for the mutual inductance from the mean distances method, we must just insert the expressions (54), (58), and (59) for the mean distances into the general equation (33):

$$
M=\frac{\mu_{0}}{2 \pi}\left[l \cdot \log (W+l)-l \cdot \log d-W+d+\frac{R^{2}}{4 d}\right]
$$

with

$$
W=\sqrt{l^{2}+d^{2}+R^{2}} .
$$

Analogously we find the correction term (48) for the Taylor series method by inserting $\Delta$ according to equation (40) and $W$ according to equation (44) and (58), which in turn also yields the above equation (63) for $W$. The final formula for the correction term is

$$
\Delta M=\frac{\mu_{0}}{2 \pi} \sqrt{d^{2}+R^{2}}\left(\sqrt{d^{2}+R^{2}}-d-\frac{R^{2}}{4 d}\right) \frac{W-l}{W(W+l)} .
$$

What we finally need is the possibility to calculate the mutual inductance exactly. Point $P_{2}$ now lies in the second cylinder whose axis through $\mathrm{O}_{2}$ is displaced from the axis through $O_{1}$ of the first cylinder by the distance $d$, see Fig. 9 . Again we use cylinder coordinates $\left(s_{1}, \psi_{1}, \mathrm{z}_{1}\right)$ with axis through $P_{2}$ for the point $P_{1}$ as shown in projection in Fig. 9.

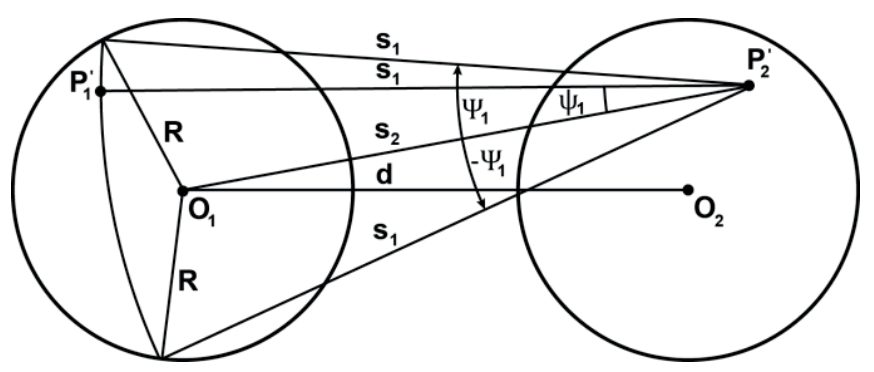

Figure 9: Polar coordinates $\left(s_{1}, \psi_{1}\right)$ for the projected integration point $P_{1}^{\prime}$. For constant $s_{1}$, the angle $\psi_{1}$ varies between $-\Psi_{1}$ and $+\Psi_{1}$.

The coordinates of the point $P_{2}$ are now $\left(s_{2}, \psi_{2}, \mathrm{z}_{2}\right)$ as shown in the projection in Fig. 10.

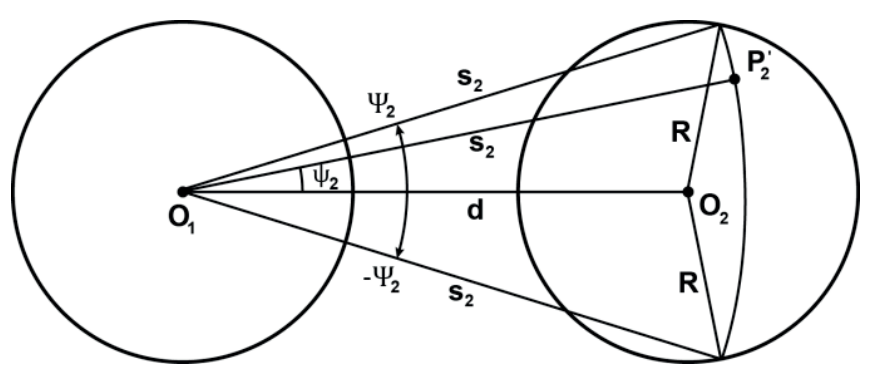

Figure 10: Polar coordinates $\left(s_{2}, \psi_{2}\right)$ for the projected integration point $P_{2}{ }^{\prime}$. For constant $s_{2}$, the angle $\psi_{2}$ varies between $-\Psi_{2}$ and $+\Psi_{2}$.

As in equations (7) and (8) we get

$$
\begin{aligned}
& \Psi_{1}=\arccos \frac{\mathrm{s}_{1}{ }^{2}+\mathrm{s}_{2}{ }^{2}-\mathrm{R}^{2}}{2 \mathrm{~s}_{1} \mathrm{~s}_{2}}=: \Psi\left(\mathrm{s}_{1}, \mathrm{~s}_{2}\right) \\
& \Psi_{2}=\arccos \frac{\mathrm{d}^{2}+\mathrm{s}_{2}{ }^{2}-\mathrm{R}^{2}}{2 \mathrm{ds}_{2}} \equiv \Psi\left(\mathrm{d}, \mathrm{s}_{2}\right) .
\end{aligned}
$$


With $\quad d \tau_{1}=s_{1} d \psi_{1} d s_{1} d z_{1} \quad, \quad d \tau_{2}=s_{2} d \psi_{2} d s_{2} d z_{2} \quad$ and $r_{12}=\sqrt{s_{1}^{2}+\left(z_{1}-z_{2}\right)^{2}}$ the integral (3) becomes

$L=\frac{\mu_{0}}{4 \pi^{3} R^{4}}$.

$\int_{0}^{l} \int_{d-R}^{d+R} \int_{-\Psi_{2}}^{\pi^{3} \Psi^{4}} \int_{0}^{l} \int_{s_{2}-R}^{s_{2}+R} \int_{-\Psi_{1}}^{\Psi_{1}} \frac{s_{1} \cdot s_{2}}{\sqrt{s_{1}^{2}+\left(z_{1}-z_{2}\right)^{2}}} d \psi_{1} d s_{1} d z_{1} d \psi_{2} d s_{2} d z_{2}$

Now the integrations over $\psi_{1}$ and $\psi_{2}$ simply yield $4 \Psi_{1}\left(s_{1}, s_{2}\right) \Psi_{2}\left(d, s_{2}\right)$ since the integrand is independent of $\psi_{1}$ and $\psi_{2}$. The integration over $z_{1}$ can be done using equation (12). Thus, again, the six-dimensional integral (3) reduces to a three-dimensional one:

$$
\begin{aligned}
& M=\frac{\mu_{0}}{\pi^{3} R^{4}} . \\
& \int_{0}^{l} \int_{d-R}^{d+R} \int_{s_{2}-R}^{s_{2}+R} \Psi\left(s_{1}, s_{2}\right) \Psi\left(d, s_{2}\right) \Lambda\left(s_{1}, z_{2}\right) s_{1} s_{2} d s_{1} d s_{2} d z_{2} .
\end{aligned}
$$

This is analogous to the integral (13) for the selfinductance. But since here the lower bounds over $s_{1}$ and $s_{2}$ are $s_{2}-R$ and $d-R$, respectively, the integral (68) is exempt from any problems of differentiability and singularity. It can directly be evaluated by means of the function integral3 for computing three-dimensional integrals in MATLAB $\AA$.

Fig. 11 shows the relative errors of the literature formula (53) of the mutual inductance between parallel wires, plotted as function of the ratio $l / R$. Three curves are shown for the parameter values $\kappa=2,3$ and 5 . The errors are considerably smaller than in the case of partial inductance as presented in section 8 .

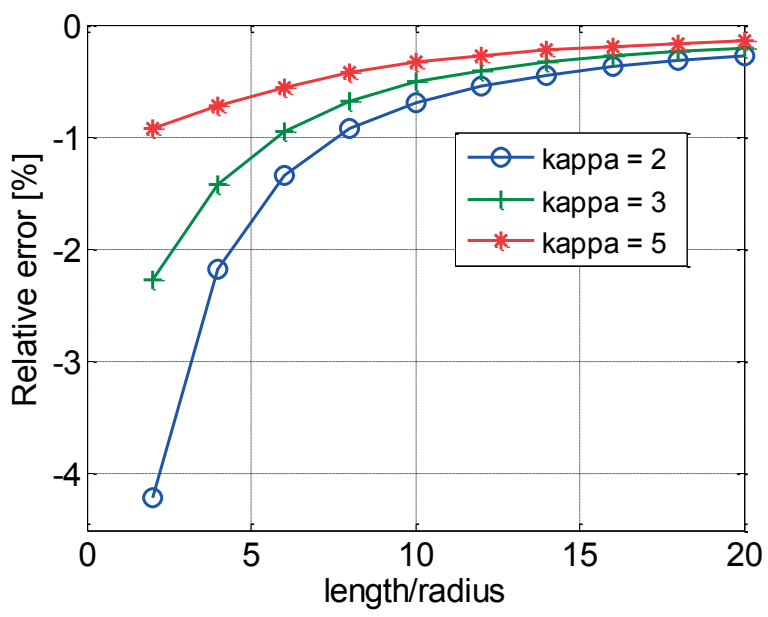

Figure 11: Relative error of the mutual inductance formula (53) from the literature plotted in function of the ratio $l / R$ for three values of the parameter $\kappa=d / R$.

Fig 12 depicts the relative errors for formula (62) derived with the mean distances method. For the larger ratios $l / R$ the curves overlap. Therefore we zoomed the plot to $l / R \geq 6$, see Fig. 13. Note that compared to Fig. 11 for small ratios $l / R$ the order of the curves in Figs. 12 and 13 is inverted. One would expect the order shown in Fig. 11, of course, because small values of $\kappa$ represent the difficult cases. Further, for the smallest ratios $l / R$ the magnitudes of the errors of equation (62) are larger than for the literature formula (53) (except for $\kappa=2$ ), despite the fact that all terms but one of equation (62) are more precise than the ones of equation (53). Further calculations with varied parameter values revealed that, strangely, for $\kappa>l / R$, i.e. $d>l$, the literature formula (53) is generally more precise than the mean distances formula (62). But for real structures the total inductance calculated with the mean distances method in these cases is still clearly more precise than with any formula from the literature because the mutual inductance of the parallel wires is much smaller than the maximal partial self-inductance occurring in the structure. For an example see Table 2 of section 10.

For all other cases, though, formula (62) is indeed more precise. Fig. 13 shows that the unexpected order of the curves completely reverts to normal for ratios $l / R>12$, so that the magnitude of the error decreases with increasing value of $\kappa$, as one would expect.

In Fig. 14 the error curves for the Taylor series method, i.e. for formulae (62) and (64) added together, are plotted. The larger the parameter $\kappa$ the smaller is the magnitude of the error, as one would expect. What is surprising here is that for small ratios, somewhere between $\kappa=2$ and $\kappa=3$, the sign of the error seems to change. The magnitudes of the errors for all values of $\kappa$ and ratios $l / R$ remain small, although they are larger than in the case of the partial selfinductance as presented in Fig. 7. Again, for practical purposes the results from the Taylor series method might be used as reference values in place of the exact values.

The main conclusions we can draw from our analysis of the mutual inductance calculations is that for $d>l$ the literature formula (53) seems to be more precise than the mean distances formula (62), although the picture changes when the total inductance of a complete structure like a shorted two-wire line or a loop coil is calculated. In all other cases equation (62) is more precise. The Taylor series method, i.e. formulae (62) and (64) added together, yields always the most accurate results.

\section{Application to a shorted two-wire line}

Although this is a theoretical paper which does not present any measurements, it might be helpful to demonstrate an application of the theory.

We take a two-wire line of length $l$, radius $R$ and distance between their centers $d$. We designate the partial selfinductance of each of the two identical wires by $L$ and their Mutual inductance by $M$. The line is shorted at one end with a wire of the same radius and of length $d$. 


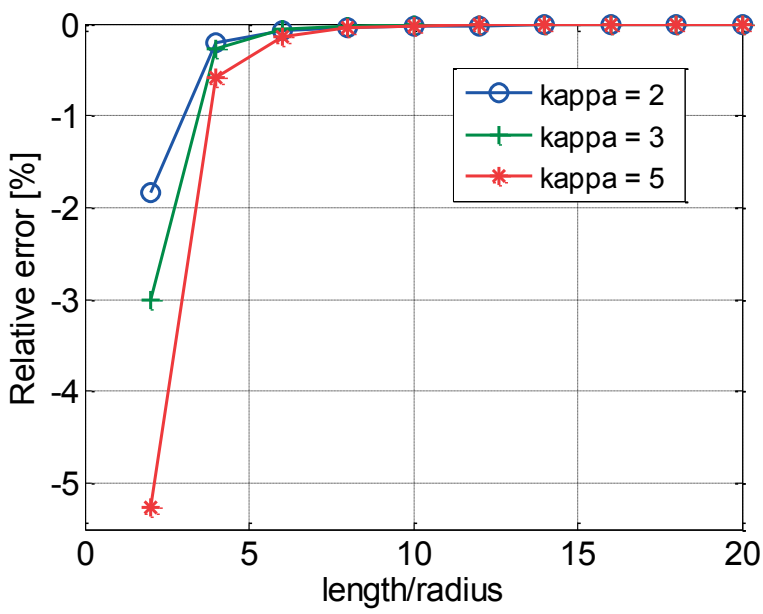

Figure 12: Relative error of the mean distances formula (62).

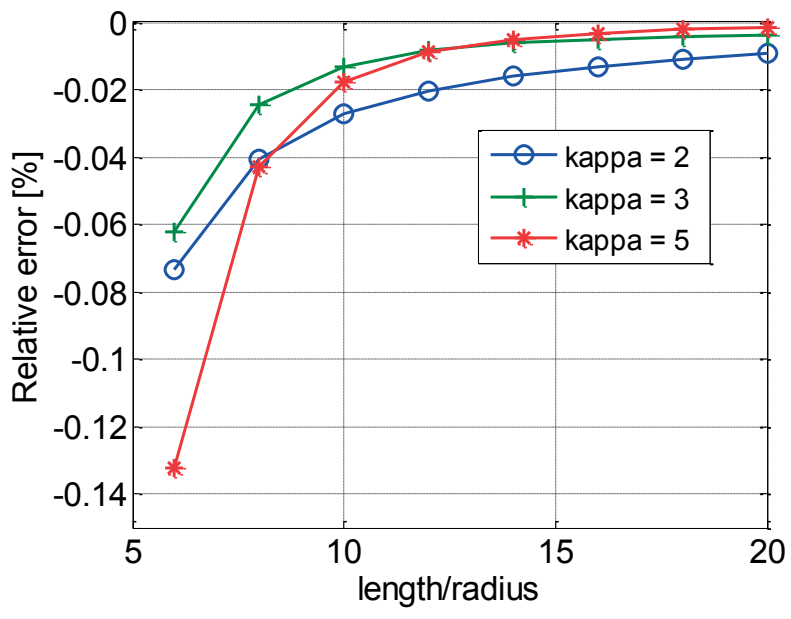

Figure 13: Same as Fig. 12, but zoomed to $l / R \geq 6$.

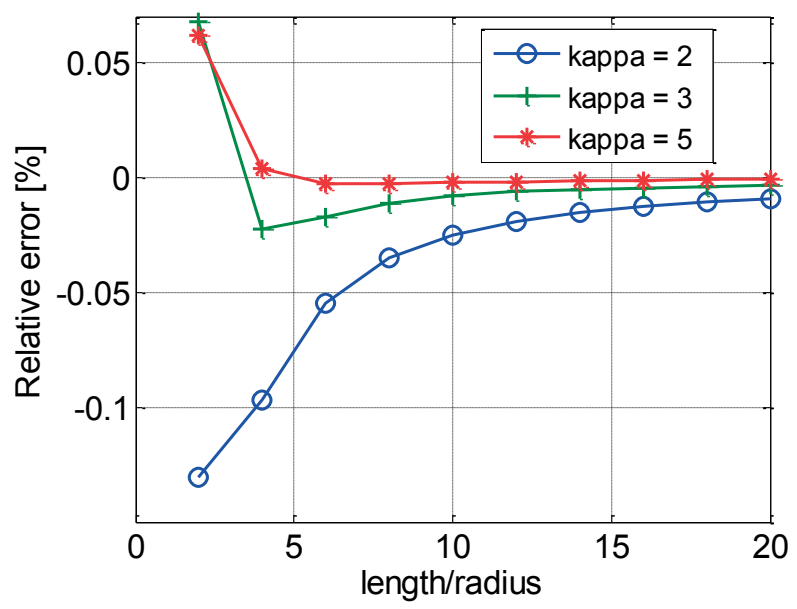

Figure 14: Relative error of formulae (62) and (64) of the Taylor series method.
Let the inductance of this shorting wire be $L_{S}$. The total self-inductance of the shorted two-wire line is then given by the equation

$$
L_{\text {total }}=2(L-M)+L_{S} .
$$

The minus sign is due to the opposite direction of current flow in the parallel wires. The results for five different twowire lines calculated with the corresponding formulae are listed in Tables 1-5. The radius of the wires is always $1 \mathrm{~mm}$. The other parameters are listed in the Tables. For $L$ and $L_{S}$ the corresponding low-frequency formulae from the previous sections were used. For the first five methods, formula (53) was used for the mutual inductance $M$. For the mean distance method, formula (62) was used, and for the Taylor series method, formulae (62) and (64) were combined.

The results show largely what one expects from the results from sections 8 and 9. The mean distances method displays a substantial improvement in accuracy over the formula from the literature; even in the unfavorable case of Table 2 where we have $\kappa=5$ and $l / R=2$ and where, according to Fig. 12, the magnitude of the error of the mutual inductance is larger than $5 \%$, compared to only $0.9 \%$ with the literature formula (see Fig. 11). But in the total structure, this error does not bear much impact on the total result because the mutual inductance $M$ is the smallest of the three terms in equation (69). It is 23 times smaller than the largest one, $L_{S}$, whose error is only $-0.002 \%$ (see Fig. 6 at $l / R=5$ ).

As expected, the improvement provided by the Taylor series method is even greater than by the mean distances method. This is particularly pronounced in the case of Table 2 where the overall accuracy of the mean distances method is reduced for the reason described above. But also the reverse effect can occur as seen in Table 4, where the Taylor series method achieves only a marginal improvement over the mean distances method. This may happen because in equation (69) terms with possibly different error signs add up.

Using the high-frequency limits in these examples would be of limited validity because one would not only need to consider the skin effect, but also the proximity effect. At the present time, no analytic description for the current distribution in close parallel wires including the proximity effect seems to be known. Once it will be known the methods described in this paper might prove helpful to develop precise analytic inductance formulae that take both skin and proximity effects into account.

Table 1: Errors of the various methods for $R=1 \mathrm{~mm}$,

\begin{tabular}{lc}
\multicolumn{2}{c}{$l=2 \mathrm{~mm}, d=2 \mathrm{~mm} . L_{\text {exact }}=0.845 \mathrm{nH}$} \\
\hline Method & Rel. error $[\%]$ \\
\hline King and Prasad & -38 \\
Meinke and Gundlach & -64 \\
Wien & -54 \\
Rosa & 17 \\
Paul & 8.5 \\
Mean distances & 0.70 \\
Taylor series & 0.086 \\
\hline
\end{tabular}


Table 2: Errors of the various methods for $R=1 \mathrm{~mm}$,

\begin{tabular}{lc}
\multicolumn{2}{c}{$l=2 \mathrm{~mm}, d=5 \mathrm{~mm} . L_{\text {exact }}=2.388 \mathrm{nH}$} \\
\hline Method & Rel. error $[\%]$ \\
\hline King and Prasad & -26 \\
Meinke and Gundlach & -29 \\
Wien & -20 \\
Rosa & 4.9 \\
Paul & 2.4 \\
Mean distances & 0.32 \\
Taylor series & 0.0021 \\
\hline
\end{tabular}

Table 3: Errors of the various methods for $R=1 \mathrm{~mm}$, $l=5 \mathrm{~mm}, d=3 \mathrm{~mm} . L_{\text {exact }}=2.785 \mathrm{nH}$.

\begin{tabular}{lc}
\hline Method & Rel. error [\%] \\
\hline King and Prasad & -48 \\
Meinke and Gundlach & -49 \\
Wien & -18 \\
Rosa & 3.9 \\
Paul & 2.6 \\
Mean distances & 0.054 \\
Taylor series & 0.012 \\
\hline
\end{tabular}

Table 4: Errors of the various methods for $R=1 \mathrm{~mm}$, $l=10 \mathrm{~mm}, d=3 \mathrm{~mm} . L_{\text {exact }}=5.401 \mathrm{nH}$.

\begin{tabular}{lc}
\hline Method & Rel. error \\
& {$[\%]$} \\
\hline King and Prasad & -53 \\
Meinke and Gundlach & -50 \\
Wien & -9.1 \\
Rosa & 2.0 \\
Paul & 1.5 \\
Mean distances & 0.0088 \\
Taylor series & 0.0077 \\
\hline
\end{tabular}

Table 5: Errors of the various methods for $R=1 \mathrm{~mm}$,

\begin{tabular}{lc}
\multicolumn{2}{c}{$l=10 \mathrm{~mm}, d=5 \mathrm{~mm} . L_{\text {exact }}=7.745 \mathrm{nH}$} \\
\hline Method & Rel. error $[\%]$ \\
\hline King and Prasad & -48 \\
Meinke and Gundlach & -44 \\
Wien & -6.6 \\
Rosa & 1.1 \\
Paul & 0.88 \\
Mean distances & 0.0071 \\
Taylor series & 0.0011 \\
\hline
\end{tabular}

\section{Conclusions}

We have shown that the known formulae for calculating the partial inductance of straight wires of circular cross section lead to large approximation errors for short wires. Since partial inductance of single wires cannot be measured, we have calculated exact numerical results as reference values which we have used to compute the relative errors of the various analytical formulae. To this end, we have presented coordinate transformations that allow reducing the defining six-dimensional integrals for the self- and the mutual inductance in the low-frequency limit of wires of circular cross section to three-dimensional ones.

We have presented two methods for deriving better analytic formulae for the partial inductance of straight wires of any cross section and for any frequency, and we have used them to derive formulae for the case of wires of circular cross section in the low- and high-frequency limits.

The methods we have presented are also valid for deriving analytic formulae for the mutual inductance of straight parallel wires of the same length, of any cross section and for any frequency. We have used them to derive analytic formulae for the mutual inductance of parallel wires of circular cross section and for low frequencies.

We have shown that for short straight wires of circular cross section the mean distances AMD and AMSD cannot be neglected, and that precise results for both the partial selfand the mutual inductance are obtained if the exact values of the AMD and AMSD are used.

We have applied our theory to some cases of short-circuited two-wire lines at low frequencies. The results show that the methods presented in this paper provide a considerable improvement in accuracy over the formulae known from the literature.

Our procedures for the exact calculation of the partial selfinductance of straight wires can easily be adapted to include skin effect, since the current distribution in circular cylinders can be calculated analytically using Bessel functions, with which the integrand is simply multiplied. As we have also presented a procedure for the exact calculation of mutual inductance in the low-frequency limit, our procedures might also prove useful for testing the accuracy of analytic formulae for the inductance of more complex structures built of straight wires, like e.g. rectangular coils. The methods developed in this paper might prove useful to develop precise analytic inductance formulae for two-wire lines and loop coils of any cross section and for any frequency, once analytic formulae for the current distribution in close parallel wires are known that take both skin and proximity effects into account.

\section{Acknowledgements}

We wish to thank Hansjörg Friedli from the Bern University of Applied Sciences for helpful discussions.

\section{References}

[1] E. B. Rosa, The self and mutual inductances of linear conductors, Bulletin of the Bureau of Standards, vol. 4, no. 2, Washington, 1907.

[2] C.R. Paul, Inductance, John Wiley \& Sons, Hoboken NJ, 2010.

[3] A. Sommerfeld, Elektrodynamik, Verlag Harry Deutsch, Frankfurt am Main, 1988, 4th ed. 2001.

[4] J. C. Maxwell, A Treatise on Electricity and Magnetism, vol. 2., Dover Publications, New York, 1954, unabridged $3^{\text {rd }}$ ed. of 1891. 
[5] F. W. Grover, Inductance Calculations, Dover Publications, New York, 2009, first published by D. Van Nostrand Co., New York, 1946.

[6] I.N. Bronstein und K.A. Semendjajew, Taschenbuch der Mathematik, Verlag Harry Deutsch, Thun, 1979.

[7] R.W.P. King and S. Prasad, Fundamental Electromagnetic Theory and Applications, Prentice Hall, Englewood Cliffs N.J., 1986.

[8] H. H. Meinke und F. W. Gundlach, Taschenbuch der Hochfrequenztechnik, Springer-Verlag, Berlin, 1992.

[9] M. Wien, Ueber die Berechnung und Messung kleiner Selbstpotentiale, Wiedemanns Annalen 53 (Annalen der Physik 289): 928-947, 1894. 